\title{
Obstructive sleep apnea: its prevention and screening may contribute to the prevention of hypertension, diabetes and cardiovascular diseases
}

\author{
Takeshi Tanigawa
}

Received: 25 January 2011 / Accepted: 8 March 2011 /Published online: 6 April 2011

(C) European Association for Predictive, Preventive and Personalised Medicine 2011

\begin{abstract}
Relationships between obstructive sleep apnea and cardiovascular diseases have been reported by both clinical and epidemiological studies. To investigate whether nocturnal intermittent hypoxia (NIH), a surrogate marker for obstructive sleep apnea in the general population, is associated with cardiovascular risk factors, we have conducted epidemiological studies in Japan. The Circulatory Risk in Communities Study (CIRCS) is a cohort study conducted across Japan to examine the risk factors for cardiovascular diseases. In CIRCS participants, NIH was estimated by the hourly occurrences of oxygen desaturation $\geq 3 \%$ ( $3 \%$ oxygen desaturation index $[3 \%$ ODI]) measured during sleep using a pulse-oximeter. Our studies showed that among middle-aged Japanese, 3\% ODI levels were associated with hypertension, elevated serum CRP levels, accumulation of metabolic risk factors, and increased risk of developing type 2 diabetes. Screening for obstructive sleep apnea in the general population is a promising and widely broadly applicable strategy for predictive, preventive and personalized medicine.
\end{abstract}

Keywords Sleep apnea - Screening · Undiagnosed · Nocturnal intermittent hypoxia $\cdot$ Cardiovascular risk factors · Epidemiology

\section{Introduction}

Obstructive sleep apnea (OSA) is a condition characterized by repetitive obstruction of the upper airway often resulting

T. Tanigawa $(\bowtie)$

Department of Public Health, Doctoral Program in Social

Medicine, Graduate School of Medicine, Ehime University,

Shitsukawa, Toon,

Ehime-ken 791-0295, Japan

e-mail: tanigawa@m.ehime-u.ac.jp in oxygen desaturation and arousal from sleep [1]. Population-based studies conducted in the United States, Europe, Australia, and Asia have shown a high prevalence of OSA. Approximately 1 in 5 adults has at least mild OSA (apnea hypopnea index $[\mathrm{AHI}] \geq 5$ ) and 1 in 15 has moderate or severe OSA (AHI $\geq 15)$ [2]. However, most patients with treatable OSA have remained undiagnosed [2]. Young and coworkers reported that $93 \%$ of women and $82 \%$ of men with moderate to severe sleep apnea syndrome have not been clinically diagnosed [3].

In patients with OSA, intermittent hypoxemia and hypercapnia during sleep, reoxygenation, intrathoracic pressure changes and arousal may cause sympathetic nervous system activation, vascular endothelial dysfunction and oxidative stress, inflammation, increased coagulation, and metabolic dysregulation [2, 4]. These factors are considered to be implicated in the development and progression of cardiovascular risk factors and diseases such as hypertension, diabetes mellitus, arrhythmias, myocardial infarction and congestive heart failure $[4,5]$.

Because of the high prevalence of OSA and the growing evidence regarding the cardiovascular consequences screening for OSA in the general population may be an important strategy for the prevention of cardiovascular diseases. This review introduces several studies conducted by our epidemiological research group in Japan regarding the association between OSA and cardiovascular risk factors.

\section{The circulatory risk in communities study (CIRCS)}

Most of our study subjects were participants in the Circulatory Risk in Communities Study (CIRCS). The CIRCS is a prospective cohort study that has been conducted in seven communities across Japan since 1963 
to examine risk factors for cardiovascular diseases, [6]. Annual cardiovascular risk surveys and monitoring of cardiovascular events were systematically conducted by a research team at the Osaka Medical Center for Health Science and Promotion, Osaka University, and the University of Tsukuba. Sleep was investigated as a part of the annual cardiovascular surveys in three communities (Ikawa; a north-east rural community, Yao; a mid-west suburban community, and Kyowa; a mid-east rural community).

\section{Nocturnal intermittent hypoxia (NIH), a surrogate marker for OSA}

In our epidemiological studies, NIH has been used as a surrogate marker for OSA. NIH was estimated by the hourly occurrences of oxygen desaturation $\geq 3 \%$ during sleep (oxygen desaturation index $\geq 3 \%$ [ $3 \%$ ODI]). Oxygen saturation was measured by a pulse-oximeter (PULSOX3Si, Minolta Co., Osaka, Japan) during a night's sleep in the participant's own home. The sensor probe was fitted to the fourth or fifth finger and secured with tape. The device calculated peripheral blood oxygen saturation values by performing a moving average of the last $5 \mathrm{~s}$, and updating each second; this sampling time was short enough to avoid underestimation of oxygen desaturation [7]. Data was downloaded to a personal computer through an interface (PULSOX IF-3, Minolta) and analyzed by proprietary software (DS-3 ver. 2.0a, Minolta). The program calculated the number of $\geq 3 \%$ desaturation events, defined as episodes beginning when oxygen saturation decreased by $3 \%$ or more for an interval of 8 to $120 \mathrm{~s}$, and terminating when the saturation rose back to baseline within a period of $20 \mathrm{~s}$. Participants recorded their sleep logs in the morning after pulse-oximetry was performed.

Because the measurement time estimated by the pulse oximeter was often longer than the true total sleep time, we used the sleep log to exclude the waking time from the analysis, thereby minimizing potential overestimation of sleep time. The 3\% ODI during the estimated sleep period $(>4 \mathrm{~h})$ was computed for each subject and used for the analysis. The validity of pulse oximetry using synchronous overnight recording of both PULSOX-3Si and standard polysomnography (PSG) was previously reported among 256 consecutive patients in a sleep-disordered breathing center (mean body mass index $[\mathrm{BMI}]=26.8 \mathrm{~kg} / \mathrm{m}^{2}$ ). The sensitivity and specificity were $80 \%$ and $95 \%$, respectively, for detecting AHI of $\geq 5$ by PSG using a cut-off threshold of $3 \% \mathrm{ODI}=5$. The sensitivity and specificity were $85 \%$ and $100 \%$, respectively, for detecting AHI of $\geq 20$ by PSG using a cut-off threshold of $3 \%$ ODI $=15$ [8]. Mild and moderate to severe NIH were defined as 3\% ODI of 5 and 15 events per hour, respectively.

\section{Risk factors for OSA}

To date, male sex, aging, craniofacial and upper airway abnormalities, and obesity have been reported as established risk factors for OSA, while genetics, smoking, menopause, alcohol use before sleep, and nighttime nasal congestion have been suggested as suspected risk factors [9]. Recently, we studied the association between alcohol consumption and OSA in community-dwelling men and women, and truck drivers. We found that alcohol consumption was associated with the severity of NIH during sleep among community-dwelling men and women, and male truck drivers [10-12]. The significant association between alcohol consumption and NIH severity suggest the need for OSA screening and alcohol intake modification as well as weight control to prevent and control OSA among both truck drivers and the general population.

To assess the impact of craniofacial morphology and body weight on OSA, we measured BMI, seven cephalometrlc variables and 3\% ODI among 313 Japanese men aged 20 to 65 years who were examined at a sleep clinic [13]. We found that the craniofacial score, derived from the distance from the midpoint of the fossa hypophysialis to the most anterior point of the frontonasal suture and the distance from the most anterosuperior point of the hyoid bone to the mandibular plane, was associated with the severity of NIH, independent of BMI. This association was stronger among overweight men than non-overweight men. Our results imply the importance of cephalometric assessment especially in overweight patients.

\section{Hypertension among community residents}

We conducted a population-based cross-sectional study of 1,424 men aged 40-69 years [14] and 3,568 women aged 30-69 years [15] who were participants in the CIRCS. The $3 \%$ ODI level was positively associated with systolic and diastolic blood pressure levels (SBP/DBP) in men; multiple regression analysis showed that a 5 event per hour increment of the $3 \%$ ODI level corresponded to a $0.8 \mathrm{mmHg}$ (95\% CI, 0.0-1.6) and $0.7 \mathrm{mmHg}$ (95\% CI, 0.3-1.1) elevation of SBP and DBP, respectively. The multivariable odds ratio for hypertension in men with moderate to severe NIH was 1.6 (95\% CI, 1.1-2.5) [14] (Table 1). This association was more evident among overweight than nonoverweight men. For women, 3\% ODI was positively associated with systolic and diastolic blood pressure levels. The multivariable odds ratios $(95 \% \mathrm{CI})$ for hypertension were $1.1(0.9-1.4), 1.2(0.8-1.8)$, and $2.2(1.4-3.4)$ in women with a $3 \%$ ODI of $5-9,10-14$, and $\geq 15$, respectively, in comparison to women with a $3 \%$ ODI $<5$ [15] (Table 1). The significant association between NIH and high blood 
Table 1 Multivariable-adjusted means (SEM) of cardiovascular risk factors and odds ratios of obstructive sleep Apnea according to 3\% ODI Level

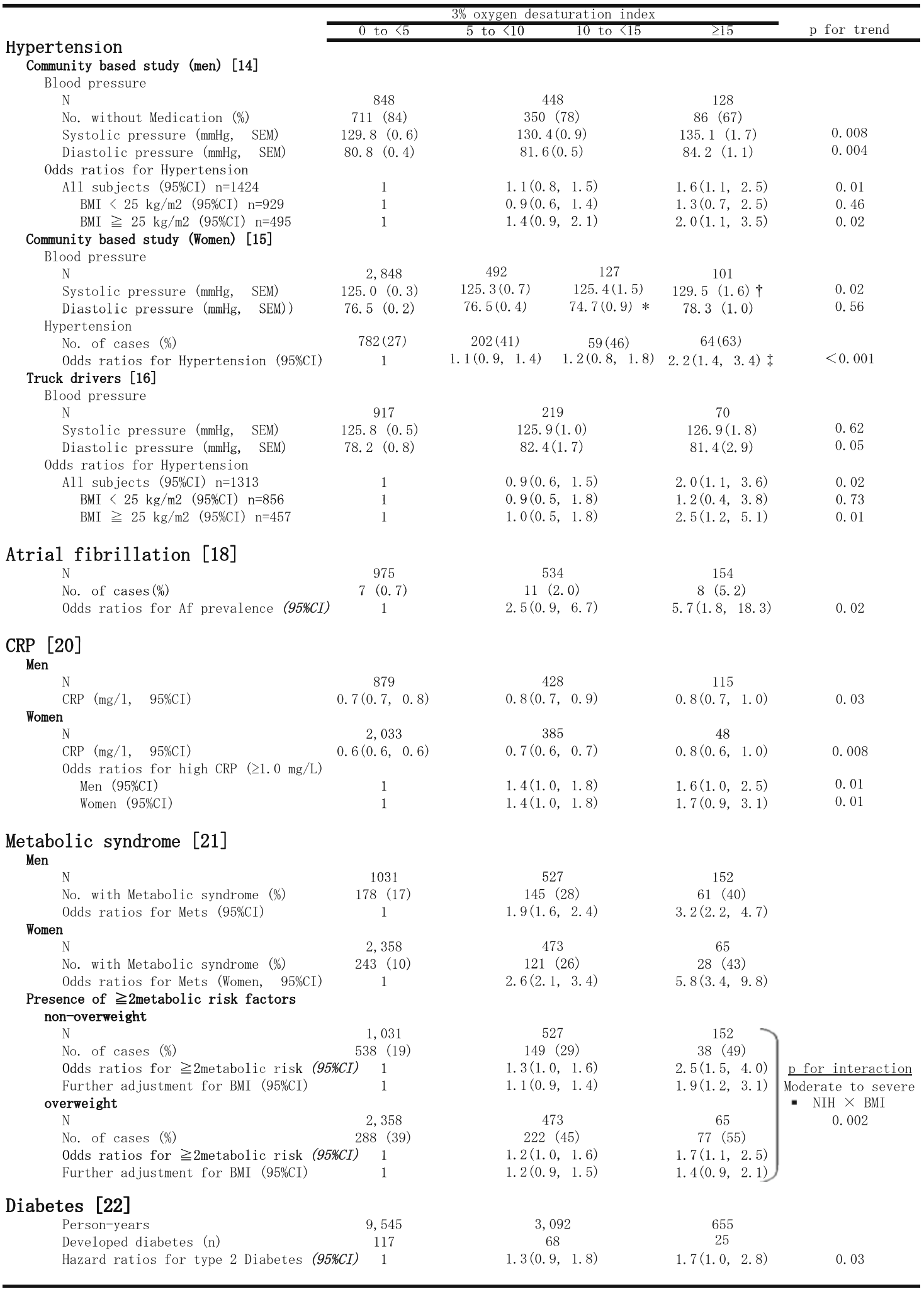

$* p<0.05, * * p<0.01, * * * p<0.001$, compared with $3 \%$ ODI $<5$ group

95\% CI: 95\% Confidence Intervals

BMI: body mass index

NIH: nocturnal intermittent hypoxia

CRP: $\mathrm{C}$ reactive protein 
pressure levels suggests that OSA plays a role in the development of hypertension in Japanese men and women.

\section{Hypertension and excessive daytime sleepiness among truck drivers}

We conducted a cross-sectional study of 1,313 truck drivers (1,258 men and 55 women aged 20-69 years), and found a significant positive association between the $3 \%$ ODI level and the diastolic blood pressure [16]. The multivariable odds ratio for hypertension was $2.0(1.1,3.6)$ for subjects with moderate to severe $\mathrm{NIH}$ in comparison to those with a $3 \%$ ODI of $<5$ (Table 1). This association was more evident among those aged $\geq 40$ years and overweight subjects. Further, the multivariable odds ratio for excessive daytime sleepiness, defined as an Epworth Sleepiness Scale score $\geq 11$ was $2.3(1.1,4.9)$ for subjects with moderate to severe $\mathrm{NIH}$ in comparison to those with a $3 \%$ ODI $<5$. This association was more evident among those aged $\geq 40$ years. The associations between NIH severity and diastolic blood pressure levels and excessive daytime sleepiness suggest the need for OSA screening among truck drivers for prevention of hypertension and potential traffic accidents.

\section{Blood pressure among shift workers}

To examine whether the relationship between OSA and blood pressure/hypertension is more evident among night shift workers than among day workers, we measured blood pressure and NIH among 253 male shift workers and 206 male day workers aged 30 to 62 years at nuclear power plants in Japan [17]. The prevalence of sleep-disordered breathing (3\% ODI $\geq 10$ and $\geq 15$ per hour) in all subjects was $11.3 \%$ (95\%CI: 8.4-13.2) and 6.1\% (95\% CI: 3.9-8.3), respectively, with no difference between shift and day workers. Diastolic blood pressure levels were significantly correlated with 3\% ODI in all subjects after adjusting for potential confounding variables $(p=0.04)$. This association was primarily observed among workers aged $\geq 40$ years, more specifically older shift workers. The correlation between NIH severity and diastolic blood pressure levels among shift workers aged $\geq 40$ years suggests the importance of screening for OSA among shift workers for blood pressure control.

\section{Atrial fibrillation among community residents}

We investigated the association between OSA and the prevalence of atrial fibrillation among 1,763 men aged 4074 years who participated in the CIRCS [18]. We found a significant association between the severity of NIH and the prevalence of atrial fibrillation; the multivariable odds ratios were 2.5 (95\% CI: 0.9-6.7) for those with mild NIH and 5.7 (95\% CI: 1.8-18.3) for moderate to severe NIH (Table 1). This study suggests that the detection of OSA and its successful treatment could be one public health approach to reduce the risk and the cost of atrial fibrillation.

\section{High-sensitivity $C$ reactive protein among community residents or workers}

We examined the relationship between serum CRP levels and $\mathrm{NIH}$, stratified by BMI category. The subjects consisted of 316 men with a mean BMI of $25.4 \mathrm{~kg} / \mathrm{m}^{2}$, aged $20-79$ years, who were examined at a sleep clinic [19]. Multivariableadjusted mean CRP levels were $0.63,0.65$, and $0.96 \mathrm{mg} / \mathrm{L}$ for NIH severity levels of $3 \%$ ODI $<5,5$ to 19.9 , and $>=20$, respectively ( $\mathrm{p}$ for trend $=0.02$ ). This association tended to be stronger in non-overweight men (BMI $\left.<25 \mathrm{~kg} / \mathrm{m}^{2}\right)(0.47$, 0.48 and $1.02 \mathrm{mg} / \mathrm{L}, \mathrm{p}$ for trend $=0.02)$ than in overweight men $\left(B M I \geq 25 \mathrm{~kg} / \mathrm{m}^{2}\right)(0.92,0.87$ and $1.21 \mathrm{mg} / \mathrm{L}$, p for trend $=0.1$ ). Thus, OSA seems to be associated with increased levels of CRP, especially in non-overweight men. Our results suggest the importance of follow-up and control of OSA in the prevention of cardiovascular diseases cannot be overlooked even in non-overweight OSA patients.

We also measured CRP and NIH in CIRCS participants 1,422 male and 2,466 female community residents aged 40-69 [20]. Multivariable-adjusted mean values of CRP among men were $0.70 \mathrm{mg} / \mathrm{L}, 0.82 \mathrm{mg} / \mathrm{L}$, and $0.84 \mathrm{mg} / \mathrm{L}$ for those with absent, mild, and moderate to severe NIH, respectively ( $p$ for trend $=0.03$ ) (Table 1 ). The values among women were $0.59 \mathrm{mg} / \mathrm{L}, 0.66 \mathrm{mg} / \mathrm{L}$, and $0.82 \mathrm{mg} / \mathrm{L}$, respectively ( $p$ for trend $=0.008$ ). The prevalence of high CRP ( $\geq 1.0 \mathrm{mg} / \mathrm{L}$ ) was 1.4 to 1.7 -fold higher for subjects of both sexes with mild to severe NIH compared to those without NIH (Table 1). OSA seems to be associated with elevated CRP levels among middle-aged Japanese, independent of atherosclerotic risk factors.

\section{Metabolic syndrome among community residents}

We examined 1,710 male and 2,896 female Japanese subjects aged 40 to 69, who participated in the CIRCS [21]. Metabolic syndrome was defined by the modified criteria of the Adult Treatment Panel III guideline. Among men, multivariable odds ratios for metabolic syndrome were 1.9 for subjects with mild NIH and 3.2 for those with moderate to severe $\mathrm{NIH}$ in comparison to those with no NIH (Table 1). Among women, the odds ratios were 2.6 and 5.8 for those with mild and moderate to severe NIH, respectively (Table 1). When stratified by BMI (non-overweight: BMI $<25 \mathrm{~kg} / \mathrm{m}^{2}$, 
overweight: BMI $\geq 25 \mathrm{~kg} / \mathrm{m}^{2}$ ), multivariable odds ratios for the presence of $\geq 2$ metabolic risk factors (other than high BMI) in those with moderate to severe NIH were 1.9 and 1.4 among non-overweight and overweight subjects, respectively (p for interaction=0.002) (Table 1). NIH was associated with the accumulation of metabolic risk factors, especially among non-overweight individuals. Our findings suggest that OSA has a stronger effect on cardiovascular risk factors in populations with a lower BMI. Therefore, the impact of OSA on cardiovascular risk factors may be large in Asia.

\section{Type 2 diabetes among community residents}

Although OSA was reported to be associated with diabetes mellitus in cross-sectional studies, a prospective association between obstructive sleep apnea and type 2 diabetes had not been clearly established [5]. We examined the association between NIH and the risk of type 2 diabetes in a prospective study [22]. A total of 4,398 community residents aged 40 to 69 years who participated in a sleep study from 2001 to 2005 were enrolled. The development of type 2 diabetes was defined by: 1) fasting serum glucose $\geq 7.00 \mathrm{mmol} / \mathrm{L}$ $(126 \mathrm{mg} / \mathrm{dL}), 2)$ non-fasting serum glucose $\geq 11.1 \mathrm{mmol} / \mathrm{L}$ $(200 \mathrm{mg} / \mathrm{dL})$ and/or 3) initiation of anti-diabetic medication or insulin therapy. By the end of 2007, follow up was completed with $92.2 \%$ of participants (median follow-up duration (interquartile range) $=3.0(2.9-4.0)$ years), and 210 subjects were found to have developed diabetes. The multivariable-adjusted hazard ratios for developing type 2 diabetes were 1.3 among those with mild NIH and 1.7 among those with moderate to severe NIH ( $p$ for trend= 0.03 ) (Table 1). NIH was associated with increased risk of developing type 2 diabetes among middle-aged Japanese, independent of traditional risk factors such as age, sex and body mass index. Furthermore, the association was not confounded by smoking or drinking behavior, the presence or absence of borderline diabetes, single night sleep duration or menopausal status in women.

\section{Perspective}

The strengths of our studies include the use of a large community-based population and the evaluation of OSA severity while the subjects sleep at home. Compared with hospital or laboratory studies, our approach has the advantage of providing a more realistic estimation of the severity of OSA in the general population because subjects are able to maintain regular daily routines with regard to sleep, physical activity, diet and ethanol intake. However, pulse-oximetry inherently underestimates respiratory disturbance events during sleep in a non-obese population compared with full polysomnography. Using polysomnography as the gold standard, previous studies report that pulse oximetry detected apneic/hypopneic events $\geq 5 / \mathrm{h}$ with a sensitivity of $68 \%$ among subjects with BMI $\leq 27.0 \mathrm{~kg} / \mathrm{m}^{2}$ and $94 \%$ for those with a BMI of $>27.0 \mathrm{~kg} / \mathrm{m}^{2}$ [8]. Pulse oximetry may be less sensitive among lean subjects because these subjects have a functional reserve of lung volume

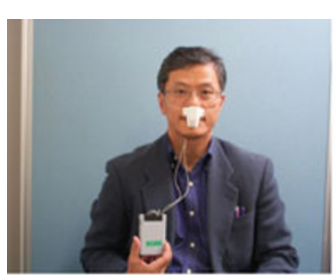

(a)

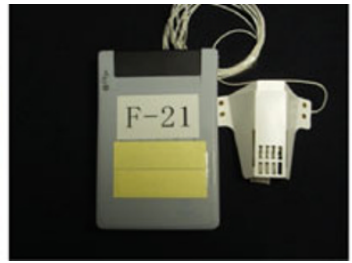

(b)

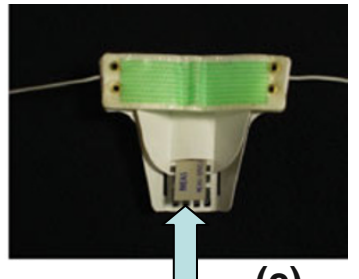

(c)

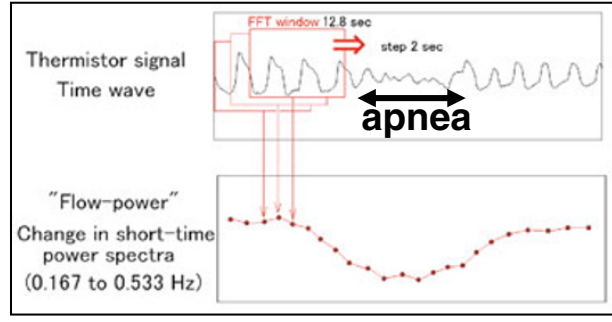

(d)

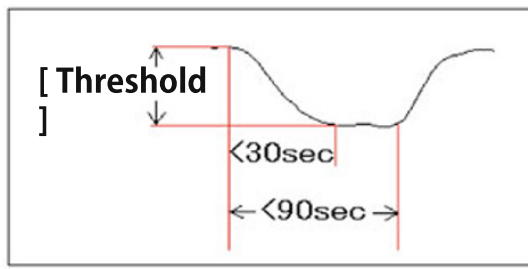

(e)

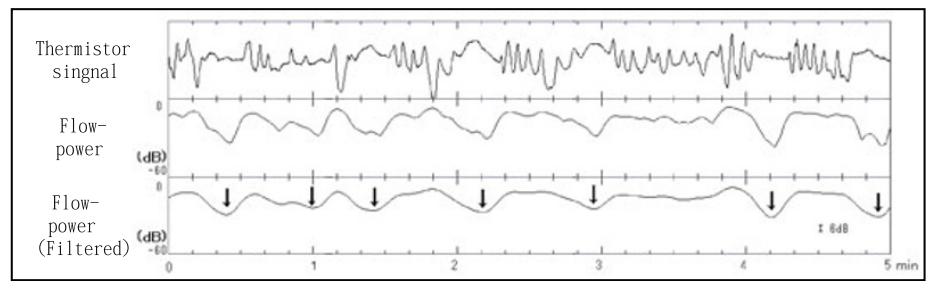

Fig. 1 The process to detect for sleep-disordered breathing by the single-channel air flow monitor using a polyvinylidene fluoride sensor. (a) The disposable polyvinylidene fluoride sensor attached below the nose. (b) The recording device and the disposable sensor. (c) The back of the sensor. The arrow indicates the polyvinylidene fluoride sensor. (d) Schematic display for signal processing in the algorithm. Procedures to obtain flow-power time series from the raw data of airflow signal are shown, which includes an apnea event. (e) Flow-power time series show a flow-power dip corresponding to an apnea/hypopnea event. A flow-power dip is defined as a fall of more than a threshold value within $30 \mathrm{~s}$, followed by a normalization part starting within $90 \mathrm{~s}$. The computer algorithm detects any decrease as a possible start-point of the flow-power dip and determines whether the following part satisfies the above conditions. (f) A 5-min flow trace in which 7 events of apnea or hypopnea are observed. Filtered flowpower shows a clear transient decrease (flow-power dip; $\downarrow$ ), which corresponding to an apnea or hypopnea event 
sufficient to maintain normal blood oxygen levels, and hypopneic events that do not cause oxygen desaturation cannot be detected by pulse oximetry.

To overcome this disadvantage, we have developed a novel screening system for OSA. The system includes a singlechannel air flow monitor using polyvinylidene fluoride (PVDF) and an original algorithm [23] developed for the analysis of thermal flow sensor signals (Fig. 1). The system determines the flow-respiratory disturbance index (flow-RDI) which shows relatively high agreement with the AHI assessed with concurrent full PSG recording. The sensitivity and specificity of the in-laboratory flow-RDI to diagnose SDB were 0.96 and $0.82,0.91$ and 0.82 , and 0.89 and 0.96 , for AHI $\geq 5, \geq 15$ and $\geq 30$ events/h, respectively. The oximetry-derived ODI agreed less closely with the AHI in normal weight subjects than in overweight subjects, but this was not the case for the flow-RDI; in non-obese subjects, the correlation between the AHI and ODI was poorer than between the AHI and flowRDI ( $r=0.87$ versus $r=0.93)$ [24]. A patent was recently granted on the automatic apnea/hypopnea detection device, detection method, software and recording medium (Japanese Patent No.4642626, December 10, 2010).

To compare the prevalence of sleep-disordered breathing among Hispanic Americans ( $n=211$, mean aged 62), white Americans $(n=246$, mean aged 62) and Japanese $(n=978$, mean aged 63), we used our single-channel airflow monitoring system [25]. In this study, sleep-disordered breathing was defined as a flow-RDI of $\geq 15$ events $/ \mathrm{h}$. The prevalence of sleep-disordered breathing was higher among Hispanics $(36.5 \%)$ and whites $(33.3 \%)$ than Japanese $(18.4 \%)$. The association of BMI with sleep-disordered breathing was strong and similar among the race/ethnic groups. Therefore, the majority of the race/ethnic difference in sleep-disordered breathing prevalence appears to be explained by the difference in BMI distribution.

We also examined the prevalence of sleep-disordered breathing among 5,320 Japanese male tracking drivers using the single-channel monitor [26]. The prevalence of sleep-disordered breathing (RDI $\geq 10$ ) was $9.1 \%$ (mean age was 44.7 , BMI was $24.8 \mathrm{~kg} / \mathrm{m}^{2}$ ), when stratified by the BMI, age-adjusted means of RDI were 5.8 (BMI $<18.5$ ), $7.2(24.9>\mathrm{BMI} \geq 18.5), 10.0(29.9>\mathrm{BMI} \geq 25)$ and 18.7 (BMI $\geq 30$ ), respectively ( $\mathrm{p}$ for trend $<0.001$ ).

\section{Concluding remarks}

Although OSA is a prevalent pathological condition, most of the patients remain undiagnosed. Recent advances in research on the cardiovascular consequences of OSA, as well as the findings of our studies have implications for the prevention and treatment of hypertension, diabetes and cardiovascular diseases. When clinicians and researchers examine the relationship between cardiovascular risk factors and the prevalence and/or incidence of cardiovascular diseases, they should consider the effects of OSA as a potential confounding factor. Furthermore, the evaluation of OSA using a pulse-oximetry and/or the single-channel air flow monitor as a noninvasive screening method is less expensive and more convenient than standard full polysomnography. Thus, these screening methods seem to be a promising and broadly applicable strategy for predictive, preventive, and personalized medicine.

Acknowledgements This study was supported in part by grants from the Japanese Ministry of Education, Culture, Sports, Science and Technology (Grant-in-Aid for research B: 22390134 in 2010-2012, Grant-in-Aid for research C: 20590634 in 2008-2010), and from the Japanese Society for the Promotion of Science, and by Health and Labour Sciences Research Grants from the Ministry of Health, Welfare and Labour, Japan.(Comprehensive Research on Life-Style Related Diseases including Cardiovascular Diseases and Diabetes Mellitus in 2010-2012).

\section{References}

1. The Report of an American Academy of Sleep Medicine Task Force. Sleep-related breathing disorders in adults: recommendations for syndrome definition and measurement techniques in clinical research. Sleep. 1999;22:667-89.

2. Somers VK, White DP, Amin R, Abraham WT, Costa F, Culebras A, et al. Sleep apnea and cardiovascular disease: an American Heart Association/American College of Cardiology Foundation scientific statement. Circulation. 2008;118:1080-111.

3. Young T, Evans L, Finn L, Palta M. Estimation of the clinically diagnosed proportion of sleep apnea syndrome in middle-aged men and women. Sleep. 1997;9:705-6.

4. Shamsuzzaman AS, Gersh BJ, Somers VK. Obstructive sleep apnea: implications for cardiac and vascular disease. JAMA. 2003;290:1906-14.

5. Shaw JE, Punjabi NM, Wilding JP, Alberti KG. International Diabetes Federation Taskforce on Epidemiology and Prevention. Sleep-disordered breathing and type 2 diabetes: a report from the International Diabetes Federation Taskforce on Epidemiology and Prevention. Diab Res Clin Pract. 2008;81: $2-12$.

6. Imano H, Kitamura A, Sato S, Kiyama M, Ohira T, Yamagishi K, et al. Trends for blood pressure and its contribution to stroke incidence in the middle-aged Japanese population: the Circulatory Risk in Communities Study (CIRCS). Stroke. 2009:40:1571-7.

7. Clark JS, Votteri B, Ariagno RL, Cheung P, Eichhorn JH, Fallat RJ, et al. Noninvasive assessment of blood gases. Am Rev Respir Dis. 1992;145:220-32.

8. Nakamata M, Kubota Y, Sakai K, Kinefuchi S, Nakayama H, Ohdaira $\mathrm{T}$, et al. The limitation of screening test for patients with sleep apnea syndrome using pulse oximetry. J Jpn Soc Respir Care. 2003;12:401-6. in Japanese.

9. Young T, Skatrud J, Peppard PE. Risk factors for obstructive sleep apnea in adults. JAMA. 2004;29:2013-6.

10. Tanigawa T, Tachibana N, Yamagishi K, Muraki I, Umesawa M, Shimamoto $\mathrm{T}$, et al. Usual alcohol consumption and arterial oxygen desaturation during sleep. JAMA. 2004;292:923-5.

11. Cui R, Tanigawa T, Sakurai S, Yamagishi K, Muraki I, Imano H, et al. Associations between alcohol consumption and sleep- 
disordered breathing among Japanese women. Respiratory Medicine. 2011 doi:10.1016/j.rmed.2011.01.003.

12. Sakurai S, Cui R, Tanigawa T, Yamagishi K, Iso H. Alcohol consumption before sleep associates the severity of sleepdisordered breathing among professional Japanese truckdrivers. Alcohol Clin Exp Res. 2007;31:2053-8.

13. Yao M, Tachibana N, Okura M, Tanigawa T, Yamagishi K, Ikeda A, et al. Relationships of craniofacial morphology and BMI with SDB in Japanese men. Laryngoscope. 2004; 114:1838-42.

14. Tanigawa T, Tachibana N, Yamagishi Y, Murakami I, Kudo M, Ohira T, et al. Relationship between sleep-disordered breathing and blood pressure levels in community-based samples of Japanese men. Hypertens Res. 2004;27:479-84.

15. Cui R, Tanigawa T, Sakurai S, Yamagishi K, Imano H, Ohira T, et al. Associations of sleep-disordered breathing with excessive daytime sleepiness and blood pressure in Japanese women. Hypertens Res. 2008;31:501-6.

16. Cui R, Tanigawa T, Sakurai S, Yamagishi K, Iso H. Relationships between sleep-disordered breathing and blood pressure and excessive daytime sleepiness among truck drivers. Hypertens Res. 2006;29:605-10.

17. Tanigawa T, Muraki I, Umesawa M, Tachibana N, Noda H, Takahashi M, et al. Sleep-disordered breathing and blood pressure levels among shift and day workers. Am J Hypertens. 2006;19:346-51.

18. Tanigawa T, Yamagishi K, Sakurai S, Muraki I, Noda H, Shimamoto T, et al. Arterial oxygen desaturation during sleep and atrial fibrillation. Heart. 2006;92:1854-5.
19. Yao M, Tachibana N, Okura M, Ikeda A, Tanigawa T, Yamagishi K, et al. Relation between sleep-disordered breathing and high-sensitivity C-reactive protein in Japanese men. Sleep. 2006;29:661-5.

20. Muraki I, Tanigawa T, Yamagishi K, Sakurai S, Ohira T, Imano H, et al. Nocturnal intermittent hypoxia and $\mathrm{C}$ reactive protein among middle-aged community residents: a cross-sectional survey. Thorax. 2010;65:523-7.

21. Muraki I, Tanigawa T, Yamagishi K, Sakurai S, Ohira T, Imano H, et al. Nocturnal intermittent hypoxia and metabolic syndrome; the effect of being overweight: the CIRCS study. J Atheroscler Thromb. 2010;17:369-77.

22. Muraki I, Tanigawa T, Yamagishi K, Sakurai S, Ohira T, Imano H, et al. Nocturnal intermittent hypoxia and the development of type 2 diabetes: the circulatory risk in communities study (CIRCS). Diabetologia. 2010;53:481-8.

23. Nakano H, Tanigawa T, Furukawa T, Nishima S. Automatic detection of sleep-disordered breathing from a single-channel airflow record. Eur Respir J. 2007;29:728-36.

24. Nakano H, Tanigawa T, Furukawa T, Ohnishi Y, Uemori H, Senzaki $\mathrm{K}$, et al. Validation of a single channel airflow monitor for screening of sleep-disordered breathing. Eur Respir J. 2008;32:1060-7.

25. Yamagishi K, Ohira T, Nakano H, Bielinski SJ, Sakurai S, Imano $\mathrm{H}$, et al. Cross-cultural comparison of the sleep-disordered breathing prevalence among Americans and Japanese. Eur Respir J. 2010;36:379-84.

26. Cui R, Tanigawa T, Nakano H, Sakurai S, Yamagishi K, Ohira T, et al. Associations between weight change since 20 years of age and sleep-disordered breathing among male truck drivers. Int J Obes (Lond). 2009;33:1396-401. 